\section{Failed intubation}

To the Editor:

The editorial responses to Davies et al. ${ }^{1}$ re-emphasize the importance of assessment, and of familiarity with any technique and equipment which the anaesthetist may use when intubation fails. The importance of learning drills for failed intubation early in postgraduate training, and for the availability of anaesthetists' assistants, ${ }^{2}$ is mentioned by all of the authors. Fear, ${ }^{3}$ in his concluding paragraph, raises the medico-legal use of published management recommendations and professional standards. He correctly indicates that, if litigation occurs, expert testimony and cross-examination will result in these discussions carrying significant weight in the evaluation of defendants' actions. It is not clear whether Fear believes this is appropriate.

The alternative to the use of published discussions of professional standards and recommendations is a defense from within a void of professional discussion about appropriate protocols. This would promote one of two extremes. First, this lack of fine-grained discussion would lead to very low standards of care being the rule for judging professional practice. Very low standards for defense of professional behaviour has the disadvantage of making challengeable the disciplinary actions of professional associations. Monitoring of professional behaviour according to vague requirements is often viewed by the courts as an illegal restriction of practice. Vague or poorly articulated standards also inhibit the improvement and development of the profession in support of the wellbeing of patients. The second extreme is that inconsistency between "medical communities" could lead to enforcement of legal rather than medical-informed definitions of what a patient should be able to expect. It is preferable for legal judgments to be based on professional discussions which discuss controversies and limitations so that individual anaesthetists are not held hostage to arbitrary standards in whose formation they have not participated.

Of greater importance, however, is the professional and ethical commitment to evaluate procedures for their effectiveness and safety. As reflected in the article and the editorials, there is controversy over the effectiveness and safety of protocols for failed intubation, and this may vary more among anaesthetists than among protocols. At the very least, such controversy and inconsistency demands careful data gathering for retrospective review, although a prospective randomized study of the protocols by trained anaesthetists would probably yield a more dependable data-base for evaluation (although this might be difficult to implement).
The possibility of litigation, and the development of carefully evaluated protocols, combine to support detailed discussions and research. Physicians' knowledge, skill, and focus on patient interest is the ethical foundation for patient trust. This is particularly true for patients under general anaesthesia who must trust anaesthetists to act proficiently and in their best interests. Discussions of appropriate protocols in professional journals and forums, evaluations of safety and efficacy and dissemination of information and skills through educational protocols are essential to justify and fulfill this trust.

Michael M. Burgess PhD

Faculty of Medicine

University of Calgary

\section{REFERENCES}

1 Davies $J M$, Weeks MB, Crone LA, Pavlin E. Failed intubation in the parturient. Can J Anaesth 1989; 36: 668-74.

2 Tunstall ME. Editorial: failed intubation in the parturient. Can J Anaesth 1989; 36: 611-3.

3 Fear DW. Editorial: failed intubation in the parturient. Can J Anaesth 1989; 36: 614-6.

\section{Pulmonary artery catheter failure}

\section{To the Editor:}

During the course of administering an anaesthetic to a 66-year-old man for resection of an abdominal aortic aneurysm, a pulmonary artery catheter was inserted (American Edwards Laboratory, 93A-131-7F). The insertion of the catheter was without incident and the catheter functioned normally during the course of surgery and immediately after transfer into the recovery room.

Two hours after the patient was admitted to the recovery room, we were informed by his nurse that the catheter has become "wedged." In fact, when reading from the distal port, a normal pulmonary artery waveform was evident. On switching to the proximal port (right atrium) only a flat line, without distinguishing features, was evident on the screen. The catheter was flushed, first with the in-line flush device, then with a heparinizedsaline loaded syringe, without change in the waveform. However, when flushing with the syringe, a stream of clear fluid was seen to exit the catheter at the $109 \mathrm{~cm}$ mark, close to the horse-tail end of the catheter. When the catheter was more closely examined a $4 \mathrm{~mm}$ linear fracture was evident at this point. The fracture was 